\title{
Common Fixed Point for Set and Single Valued Functions without Continuity and Compatibility
}

\author{
Bhavana Deshpande
}

\begin{abstract}
We prove some common fixed point theorems for set and single valued mappings without assuming continuity and compatibility. We show that completeness of the whole space is not necessary for existence of common fixed point. To prove the theorems we use a new noncompatible condition that is weak commutativity of type $(K B)$. We also prove a fixed point theorem for sequence of set valued mappings.
\end{abstract}

\section{INTRODUCTION}

There exists an extensive literature on common fixed point of set valued mappings satisfying contractive conditions controlled by a nonnegative real valued function from $[0, \infty]$ to $[0, \infty)$. In these results suitable conditions on the control function are crucial for the existence of fixed points. For this kind of work one can be referred to Singh \& Meade [17], Barcz [1], Khan \& Kubiaczyk [7].

Sessa [15] introduced the concept of weakly commuting maps. Jungck [5] defined the notion of compatible maps in order to generalize the concept of weak commutativity and showed that weakly commuting mappings are compatible but the converse is not true. These concepts have been immediately extended to set valued maps $([3,6])$ and define what they call weak commutativity and compatibility. Fixed point theorems for set and single valued mappings have numerous applications in mathematical sciences and engineering (e.g. Kyzyska and Kubiaczyk [9], Sessa and Khan [16]). Number of these theorems are very useful but their hypothesis are very difficult to satisfy as they require continuity and compatibility of involved mappings. There are so many functions which are not continuous but has a fixed point.

2000 Mathematics Subject Classification. Primary: 47H10, 54H25.

Key words and phrases. Common fixed point, noncompatible maps, weak commutativity of type $(K B)$. 
For example the function $f$ defined on $R$ by

$$
\begin{array}{ll}
f(x)=0, & x \leq 0, \\
f(x)=1, & x>0 .
\end{array}
$$

This function $f$ is not continuous at 0 but has 0 as a fixed point.

Another example is Dirichlet function defined on $R$ by

$$
\begin{array}{ll}
f(x)=1 & \text { if } x \text { is rational, } \\
f(x)=0 & \text { if } x \text { is irrational. }
\end{array}
$$

Dirichlet function is not continuous at any point but has 1 as a fixed point.

These observations motivated several authors of the field to prove fixed point theorems for noncompatible, discontinuous mappings.

Pant $[10,11,12,13]$ initiated the study of noncompatible maps and introduced pointwise $R$-weak commutativity of mappings in [10]. He also showed that pointwise $R$-weak commutativity is a necessary, hence minimal condition for the existence of a common fixed point of contractive type maps [11].

Pathak, Cho and Kang [14] introduced the concept of $R$-weakly commuting mappings of type $A$ and showed that they are not compatible. Recently, I. Kubiaczyk and Bhavana Deshpande [8] extended the concept of $R$-weakly commutativity of type $A$ for single valued mappings to set valued mappings and introduced weak commutativity of type $(K B)$.

The purpose of this paper is to obtain some common fixed point theorems for set valued and single valued mappings defined on a metric space employing a new noncompatible condition that is weak commutativity of type $(K B)$. We show that continuity of any mapping is not necessary for the existence of common fixed point. We also show that completeness of the whole space can be replaced by a weaker condition. We improve and generalize the results of Imdad and Ahmad [4], Khan and Kubiaczyk [7] and others.

\section{Preliminaries}

In the sequel $(X, d)$ denotes a metric space and $B(X)$ is the set of all non empty bounded subsets of $X$. As in $[2,3]$ we define

$$
\begin{aligned}
\delta(A, B) & =\sup \{d(a, b): a \in A, b \in B\}, \\
D(A, B) & =\inf \{d(a, b): a \in A, b \in B\}, \\
H(A, B) & =\inf \left\{r>0: A_{r} \supset B, B_{r} \supset A\right\},
\end{aligned}
$$


for all $A, B$ in $B(X)$, where

$$
\begin{aligned}
& A_{r}=\{x \in X: d(x, a)<r \quad \text { for some } a \in A\}, \\
& B_{r}=\{y \in X: d(y, b)<r \quad \text { for some } b \in B\} .
\end{aligned}
$$

If $A=\{a\}$ for some $a \in A$, we denote $\delta(a, B), D(a, B)$ and $H(a, B)$ for $\delta(A, B), D(A, B)$ and $H(A, B)$ respectively. Also, if $B=\{b\}$ and $A=\{a\}$, one can deduce that $\delta(A, B)=D(A, B)=H(A, B)=d(a, b)$.

It follows immediately from the definition of $\delta(A, B)$ that

$$
\begin{aligned}
& \delta(A, B)=\delta(B, A) \geq 0, \quad \delta(A, B) \leq \delta(A, C)+\delta(C, B) \\
& \delta(A, B)=0 \text { iff } A=B=\{a\}, \quad \delta(A, A)=\operatorname{diam} A,
\end{aligned}
$$

for all $A, B, C \in B(X)$.

Definition $2.1([3])$. A sequence $\left\{A_{n}\right\}$ of nonempty subsets of $X$ is said to be convergent to a subset $A$ of $X$ if

(i) Each point $a$ in $A$ is the limit of a convergent sequence $\left\{a_{n}\right\}$, where $a_{n}$ is in $A_{n}$ for all $n \in N$.

(ii) For arbitrary $\epsilon>0$, there exists an integer $m$ such that $A_{n} \subseteq A_{\epsilon}$ for $n>m$, where $A_{\epsilon}$ denotes the set of all points $x$ in $X$ for which there exists a point $a$ in $A$, depending on $x$, such that $d(x, a)<\epsilon$.

$A$ is said to be the limit of the sequence $\left\{A_{n}\right\}$.

Lemma 2.1 ([3]). If $\left\{A_{n}\right\}$ and $\left\{B_{n}\right\}$ are sequences in $B(X)$ converging to $A$ and $B$ in $B(X)$, respectively, then the sequence $\left\{\delta\left(A_{n}, B_{n}\right)\right\}$ converges to $\delta(A, B)$.

Lemma $2.2([3])$. Let $\left\{A_{n}\right\}$ be a sequence in $B(X)$ and $y$ be a point in $X$ such that $\delta\left(A_{n}, y\right) \rightarrow 0$. Then the sequence $\left\{A_{n}\right\}$ converges to the set $\{y\}$ in $B(X)$.

Definition $2.2([3])$. The mappings $F: X \rightarrow B(X)$ and $f: X \rightarrow X$ are said to be weakly commuting if $f F x \in B(X)$ and

$$
\delta(F f x, f F x) \leq \max \{\delta(f x, F x), \operatorname{diam} f F x\} \quad \text { for all } x \text { in } X .
$$

Note that if $F$ is single valued mapping then the set $\{f F x\}$ consists of a single point. Therefore, $\operatorname{diam} f F x=0$ for all $x$ in $X$ and above inequality reduces to the well known condition given by Sessa [14]; that is

$$
d(F f x, f F x) \leq d(f x, F x) \text { for all } x \text { in } X .
$$

Two commuting mappings $F$ and $f$ are weakly commuting but the converse is not true as shown in [3]. 
Definition 2.3 ([5]). The mappings $F: X \rightarrow B(X)$ and $f: X \rightarrow X$ are $\delta$-compatible if $\lim _{n \rightarrow \infty} \delta\left(F f x_{n}, f F x_{n}\right)=0$ whenever $\left\{x_{n}\right\}$ is a sequence in $X$ such that $f F x_{n} \in B(X), F x_{n} \rightarrow\{t\}, f x_{n} \rightarrow t$ for some $t$ in $X$.

Definition 2.4 ([10]). The mappings $f, g: X \rightarrow X$ will be called $R$-weakly commuting, provided there exists some positive real number $R$ such that

$$
d(f g x, g f x) \leq R d(f x, g x)
$$

for each $x$ in $X . f$ and $g$ will be called $R$-weakly commuting at a point $x$ if

$$
d(f g x, g f x) \leq R d(f x, g x) \quad \text { for some } R>0 .
$$

Definition 2.5 ([14]). The mappings $f, g: X \rightarrow X$ are said to be $R$-weakly commuting of type $\left(A_{f}\right)$ if there exists a positive real number $R$ such that

$$
d(f g x, g g x) \leq R d(f x, g x) \text { for all } x \in X .
$$

Definition 2.6 ([12]). The mappings $f, g: X \rightarrow X$ are said to be $R$-weakly commuting of type $\left(A_{g}\right)$ if there exists a positive real number $R$ such that

$$
d(g f x, f f x) \leq R d(f x, g x) \text { for all } x \in X .
$$

Remark 2.1 ([14]). $\quad$ (i) Compatible mappings are $R$-weakly commuting mappings of type $\left(A_{f}\right)$ or type $\left(A_{g}\right)$ but converse is not true,

(ii) $R$-weakly commuting mappings are not necessarily $R$-weakly commuting of type $\left(A_{f}\right)$ or $R$-weakly commuting of type $\left(A_{g}\right)$.

Definition $2.7([8])$. The mappings $f: X \rightarrow X$ and $F: X \rightarrow B(X)$ are said to be weakly commuting of type $(K B)$ at $x$ if there exists some positive real number $R$ such that

$$
\delta(f f x, F f x) \leq R \delta(f x, F x) .
$$

Here $f$ and $F$ are weakly commuting of type $(K B)$ on $X$ if above inequality holds for all $x \in X$. If $f$ is single valued self mappings of $X$ the definition of weak commutativity of type $(K B)$ reduces to Definition 2.6.

Example 2.1. Let $X=[1,20]$ and $d$ be the usual metric on $X$. Define $f: X \rightarrow X$ and $F: X \rightarrow B(X)$ by

$$
\begin{gathered}
f(x)= \begin{cases}x, & \text { if } 1 \leq x \leq 5, \\
\frac{x+3}{4}, & \text { if } 5<x \leq 20 .\end{cases} \\
F x= \begin{cases}{[1, x],} & \text { if } 1 \leq x \leq 2, \\
{[2, x],} & \text { if } 2<x \leq 5, \\
{\left[2, \frac{x-1}{2}\right],} & \text { if } 5<x \leq 20 .\end{cases}
\end{gathered}
$$


Let $x_{n}=5+\frac{1}{n}, n=1,2, \ldots$ then

$$
\lim _{n \rightarrow \infty} f x_{n}=2 \text { and } \lim _{n \rightarrow \infty} F x_{n}=\{2\} .
$$

Also

$$
f F x_{n} \in B(X) \quad \text { and } \quad \delta\left(F f x_{n}, f F x_{n}\right)=\delta\left(\left[2,2+\frac{1}{4 n}\right],\left[2,2+\frac{1}{2 n}\right]\right) \rightarrow 0
$$

as $n \rightarrow \infty$. So the pair $\{F, f\}$ is $\delta$-compatible.

On the other hand if we take $x=3$ then $f f x=3, F f x=[2,3]$ and clearly $f$ and $F$ are weakly commuting of type $(K B)$ at $x=3$.

Example 2.2. Let $X=[1, \infty)$ and $d$ be the usual metric on $X$. Define $f: X \rightarrow X$ and $F: X \rightarrow B(X)$ by

$$
f(x)=4 x \text { and } F x=[1, x] \text { for all } x \in X .
$$

Then $f f x=16 x, F f x=[1,4 x]$ and for $R>5$ we can see that $\delta(f f x, F f x)<$ $R \delta(f x, F x)$ for all $x \in X$. Thus $f$ and $F$ are weakly commuting of type $(K B)$ on $X$ but there exists no sequence $\left\{x_{n}\right\}$ in $X$ such that condition of $\delta$-compatibility is satisfied.

In accordance with [7], let $\Phi$ be the set of all real valued function $\phi$ : $\left(R^{+}\right)^{5} \rightarrow R^{+}$which are upper semicontinuous from the right and and non decreasing in each of the co-ordinate variable such that $\phi(t, t, t, a t, b t)<t$ for each $t \geq 0, a \geq 0, b \geq 0$ with $a+b \leq 4$. Also $\Psi$ is the set of real valued functions $\psi: R^{+} \rightarrow R^{+}$which are upper semicontinuous from the right and nondecreasing with $\psi(t)<t$ for $t>0$.

We also require the following lemma due to Singh and Meade [17].

Lemma 2.3. For $t>0, \lim \psi^{n}(t)=0$.

\section{Main Results}

Theorem 3.1. Let $F, G$ be two set valued mappings of a metric space $(X, d)$ into $B(X)$ and $f, g$ two self mappings of $X$. Suppose the pairs $\{F, f\}$ and $\{G, g\}$ are weakly commuting of type $(K B)$ on $X$, further

$$
F(X) \subseteq(X), \quad G(X) \subseteq(X),
$$

and for all $x, y$ in $X$ and $\phi \in \Phi$

(1) $\delta(F x, G y) \leq \phi(\delta(f x, F x), \delta(g y, G y), \delta(f x, G y), \delta(g y, F x), d(f x, g y))$

where $\psi \in \Psi, t>0, a \geq 0, b \geq 0, a+b \leq 4$.

$$
\Psi(t)=\max \{\phi(t, t, t, a t, b t), \phi(t, 0,0, t, 0), \phi(0,0, t, t, t), \phi(0, t, t, 0,0)\}<t .
$$

If one of $f(X)$ or $g(X)$ is complete then $F, G, f$ and $g$ have a unique common fixed point $z$ in $X$ such that $\{f z\}=\{g z\}=\{z\}=F z=G z$. 
Proof. Let $x_{0} \in X$ and $y_{1}$ be an arbitrary point chosen in $Z_{1}=F x_{0}$. Since $F(X) \subseteq g(X)$, we get a point $x_{1} \in X$ such that $g x_{1}=y_{1}$. Now choose an arbitrary point $y_{2}$ in $Z_{2}=G x_{1}$; as $G(X) \subseteq f(X)$, we get an $x_{2} \in X$ with $f x_{2}=y_{2}$. Thus in general if we choose $x_{2 n}$ in $X$ with $y_{2 n+1} \in Z_{2 n+1}=F x_{2 n}$ then we always get some $x_{2 n+1} \in X$ satisfying $g x_{2 n+1}=y_{2 n+1}$. Again let $y_{2 n+2} \in Z_{2 n+2}=G x_{2 n+1}$ be arbitrary then there exists $Z_{2 n+2} \in X$ such that $f x_{2 n+2}=y_{2 n+2}$ for $n=0,1,2, \ldots$ Let us put $V_{n}=\delta\left(Z_{n}, Z_{n+1}\right)$. We distinguish two cases:

Case 1. If $V_{1}=0$ then

$$
V_{1}=\delta\left(Z_{1}, Z_{2}\right)=\delta\left(F x_{0}, G x_{1}\right)=0,
$$

which means that $F x_{0}=\left\{y_{1}\right\}=\left\{g x_{1}\right\}=\left\{y_{2}\right\}=\left\{f x_{2}\right\}$ and since the pair $\{G, g\}$ is weakly commuting of type $(K B)$ on $X$, therefore

$$
\delta\left(g g x_{1}, G g x_{1}\right) \leq R \delta\left(g x_{1}, G x_{1}\right) .
$$

Thus

$$
\left\{g g x_{1}\right\}=G g x_{1}=g G x_{1}=G G x_{1} .
$$

Now using (1), we get

$$
\begin{aligned}
\delta\left(F x_{2}, G x_{1}\right) & \left.\leq \phi\left(F x_{2}, G x_{1}\right), 0,0, \delta\left(F x_{2}, G x_{1}\right), 0\right) \\
& \leq \psi\left(\delta\left(F x_{2}, G x_{1}\right)\right) \\
& <\delta\left(F x_{2}, G x_{1}\right)
\end{aligned}
$$

which gives $F x_{2}=G x_{1}$.

Since $\{F, f\}$ is weakly commuting pair of type $(K B)$ on $X$, therefore

$$
\delta\left(f f x_{2}, F f x_{2}\right) \leq R \delta\left(f x_{2}, F x_{2}\right),
$$

which gives

$$
\left\{f f x_{2}\right\}=F f x_{2}=F F x_{2}=f F x_{2} .
$$

Applying (1) again we can have

$$
\begin{aligned}
\delta\left(F F x_{2}, F x_{2}\right) & =\delta\left(F F x_{2}, G x_{1}\right) \\
& <\phi\left(0,0, \delta\left(F F x_{2}, F x_{2}\right), \delta\left(F F x_{2}, F x_{2}\right), \delta\left(F F x_{2}, F x_{2}\right)\right) \\
& \leq \psi\left(\delta\left(F f X_{2}, F x_{2}\right)\right) \\
& <\delta\left(F F x_{2}, F x_{2}\right),
\end{aligned}
$$


obtaining there by $F F x_{2}=F x_{2}$. Thus $F x_{2}$ is a fixed point of $F$. It follows from (3) that $F x_{2}$ is also a fixed point of $f$. Since $F x_{2}=G x_{1}$, we can get

$$
\begin{aligned}
\delta\left(G x_{1}, G G x_{1}\right) & =\delta\left(F x_{2}, G G x_{1}\right) \\
& \leq \phi\left(0,0, \delta\left(G x_{1}, G G x_{1}\right), \delta\left(G x_{1}, G G x_{1}\right), \delta\left(G x_{1}, G G x_{1}\right)\right) \\
& \leq \psi\left(\delta\left(G x_{1}, G G x_{1}\right)\right) \\
& <\delta\left(G x_{1}, G G x_{1}\right),
\end{aligned}
$$

which gives that $G G x_{1}=G x_{1}$. Thus $F x_{2}=G x_{1}$ is a fixed point of $G$ and from (2) it follows that $F x_{2}=G x_{1}$ is also a fixed point of $g$. Thus $F x_{2}$ is a common fixed point of $F, G, f$ and $g$.

Case 2. Suppose that $V_{n}>0, n=1,2, \ldots$ then

$$
\begin{aligned}
V_{2 n+1} & =\delta\left(Z_{2 n+1}, Z_{2 n+2}\right)=\delta\left(F x_{2 n}, G x_{2 n+1}\right) \\
& \leq \phi\left(V_{2 n}, V_{2 n+1}, V_{2 n}+V_{2 n+1}, 2 V_{2 n}, V_{2 n+l}\right) .
\end{aligned}
$$

Let us assume that $V_{2 n+1}>V_{2 n}$, then

$$
V_{2 n+1} \leq \phi\left(V_{2 n+1}, V_{2 n+1}, 2 V_{2 n+l}, 2 V_{2 n+1}, V_{2 n+1}\right) \leq \psi\left(V_{2 n+1}\right)<V_{2 n+1},
$$

which is a contradiction. Hence $V_{2 n+1} \leq V_{2 n}$. Similarly one can show that $V_{2 n+2} \leq V_{2 n+1}$. Then $\left\{V_{n}\right\}$ is a decreasing sequence. Now since

$$
V_{2} \leq \phi\left(V_{l}, V_{1}, V_{1}, 2 V_{1}, 2 V_{1}\right) \leq \psi\left(V_{1}\right),
$$

it follows by induction that

$$
V_{2 n+1} \leq \psi^{2 n}\left(V_{1}\right)
$$

and hence Lemma 2.3 gives that

$$
\lim _{n \rightarrow \infty} V_{n}=0 .
$$

We now show that $\left\{y_{n}\right\}$ is a Cauchy sequence. For this it is sufficient to show that $\left\{y_{2 n}\right\}$ is a Cauchy sequence. Suppose $\left\{y_{2 n}\right\}$ is not Cauchy sequence. Then there is an $\epsilon>0$ such that for an even integer $2 k$ there exists even integer $2 m(k)>2 n(k)>2 k$ such that

$$
d\left(y_{2 n(k)}, y_{2 m(k)}\right)>\epsilon \text {. }
$$

For every even integer $2 k$, let $2 m(k)$ be the least positive integer exceeding $2 n(k)$ satisfying (4) and such that

$$
d\left(y_{2 n(k)}, y_{2 m(k)-2}\right)<\epsilon .
$$

Now

$$
\begin{aligned}
\epsilon & \leq d\left(y_{2 n(k)}, y_{2 m(k)}\right) \leq \\
& \leq d\left(y_{2 n(k)}, y_{2 m(k)-2)}+V_{2 m(k)-2}+V_{2 m(k)-1} .\right.
\end{aligned}
$$


Then by (4) and (5) it follows that

$$
\lim _{k \rightarrow \infty} d\left(y_{2 n(k)}, y_{2 m(k)}\right)=\epsilon .
$$

Also by triangle inequality, we have

$$
\left|d\left(y_{2 n(k)}, y_{2 m(k)-1}\right)-d\left(y_{2 n(k)}, y_{2 m(k)}\right)\right|<V_{2 m(k)-1},
$$

and

$$
\left|d\left(y_{2 n(k)+1}, y_{2 m(k)-1}\right)-d\left(y_{2 n(k)}, y_{2 m(k)}\right)\right|<V_{2 m(k)-1}+V_{2 n(k)} .
$$

By using (6) we get $d\left(y_{2 n(k)}, y_{2 m(k)-1}\right) \rightarrow \epsilon$ and $d\left(y_{2 n(k)+1}, y_{2 m(k)-1}\right) \rightarrow \epsilon$ as $k \rightarrow \infty$. Now by (1), we get

$$
\begin{aligned}
d\left(y_{2 n(k)}, y_{2 m(k)}\right) \leq & V_{2 n(k)}+ \\
\leq & \left(F x_{2 n(k)}, G x_{2 m(k)-1}\right) \\
\leq & V_{2 n(k)}+\phi\left(V_{2 n(k)}, V_{2 m(k)-1}, d\left(y_{2 m(k)}, y_{2 m(k)-1}\right), V_{2 m(k)-1},\right. \\
& \left.\quad d\left(y_{2 m(k)-1}, y_{2 n(k)+1}\right), V_{2 n(k)}, d\left(y_{2 n(k)}, y_{2 m(k)-1}\right)\right) .
\end{aligned}
$$

which on letting $k \rightarrow \infty$ reduces to

$$
\epsilon<\phi(0,0, \epsilon, \epsilon, \epsilon)<\epsilon,
$$

giving a contradiction. Thus $\left\{y_{2 n}\right\}=\left\{f x_{2 n}\right\}$ is a Cauchy sequence in $X$ and hence any subsequence thereof is a Cauchy sequence in $X$. Suppose $f(X)$ is complete. Then $f x_{2 n} \rightarrow z=f v \in f(X)$ for $v \in X$. But $g x_{2 n+1}=y_{2 n+1} \in$ $F x_{2 n}$. So we have $g x_{2 n+1} \in z$. Moreover we have for $n=0,1,2, \ldots$

$$
\delta\left(F x_{2 n}, z\right) \leq \delta\left(F x_{2 n}, f x_{2 n}\right)+\delta\left(f x_{2 n}, z\right) .
$$

Therefore $\delta\left(F x_{2 n}, z\right) \rightarrow 0$.

Similarly $\delta\left(G x_{2 n+1}, z\right) \rightarrow 0$.

By (1) for $n=1,2, \ldots$ we have

$$
\begin{gathered}
\delta\left(F v, G x_{2 n+1}\right) \leq \phi\left(\delta(f v, F v), \delta\left(g x_{2 n+1}, G x_{2 n+1}\right), \delta\left(f v, G x_{2 n+1}\right),\right. \\
\left.\delta\left(g x_{2 n+1}, F v\right), d\left(f v, g x_{2 n+1}\right)\right) .
\end{gathered}
$$

Letting $n \rightarrow \infty$ and using Lemma 2.1 and Lemma 2.2, we obtain

$$
\begin{aligned}
\delta(F v, z) & \leq \phi(\delta(F v, z), 0,0, \delta(F v, z), 0) \\
& \leq \psi(\delta(F v, z))<\delta(F v, z),
\end{aligned}
$$


which gives $F v=\{z\}=\{f v\}$. But $F(X) \subset g(X)$, there exists $u \in X$ such that $\{g u\}=F v=\{z\}$. Now if $F v \neq G u, \delta(F v, G u) \neq 0$ so by (1), we have

$$
\begin{aligned}
\delta(F v, G u) & \leq \phi(0, \delta(F v, G u), \delta(F v, G u), 0,0) \\
& \leq \psi(\delta(F v, G u))<\delta(F v, G u),
\end{aligned}
$$

which gives $F v=\{f v\}=\{g u\}=G u=\{z\}$. Since $F v=\{f v\}$ and the pair $\{F, f\}$ is weakly commuting of type $(K B)$ on $X$ so we have

$$
\delta(f f v, F f v) \leq R \delta(f v, F v),
$$

and so $\{f z\}=F z$.

Again since $G u=\{g u\}$ and the pair $\{G, g\}$ is weakly commuting of type $(K B)$ so we have

$$
\delta(g g u, G g u) \leq R \delta(g u, G u),
$$

which implies $\{g z\}=G z$. By (1), we have

$$
\begin{aligned}
\delta(F z, z) & \leq \delta(F z, G u) \\
& \leq \phi(0,0, \delta(F z, z), \delta(F z, z), \delta(F z, z)) \\
& \leq \psi(\delta(F z, z))<\delta(F z, z),
\end{aligned}
$$

which gives $F z=\{z\}$. Consequently, we have $\{z\}=F z=\{f z\}$. Similarly $\{z\}=G z=\{g z\}$. Therefore we have $\{z\}=\{f z\}=\{g z\}=F z=G z$. Finally, we prove that $z$ is unique. If not let $w$ be another common fixed point such that $z \neq w$ and $\{w\}=\{f w\}=\{g w\}=F w=G w$. By (1), we have

$$
\begin{aligned}
d(z, w) & \leq \delta(F z, G w) \\
& \leq \phi(0,0, d(z, w), d(z, w), d(z, w)) \\
& \leq \psi(d(z, w))<d(w, z),
\end{aligned}
$$

which gives $w=z$. This completes the proof.

Remark 3.1. Theorem 3.1 improves and generalizes the result of Imdad and Ahmad [4], Theorem 3.1 improves, extends and generalizes the result of Khan \& Kubiaczyk [7].

Theorem 3.2. Theorem 3.1 holds good if we replace the condition (1) by

$$
\delta(F x, G y) \leq \phi(\delta(f x, F y), \delta(g y, G y), D(f x, G y), D(g y, F x), d(f x, g y)) .
$$

Remark 3.2. The condition required for the constants $a$ and $b$ in Theorem 3.2 is merely $a+b \leq 2$.

If we put $f=g$ in Theorem 3.1, we get the following: 
Corollary 3.1. Let $F, G$ be two set valued mappings of a metric space $(X, d)$ into $B(X)$ and $f$ a self mapping of $X$. Suppose the pairs $\{F, f\}$ and $\{G, f\}$ are weakly commuting of type $(K B)$ on $X$, further

$$
F(X) \subseteq(X), \quad G(X) \subseteq f(X),
$$

and for all $x, y$ in $X$ and $\phi \in \Phi$

$$
\delta(F x, G y) \leq \phi(\delta(f x, F x), \delta(f y, G y), \delta(f x, G y), \delta(f y, F x), d(f x, g y))
$$

where $\psi \in \Psi, t>0, a \geq 0, b \geq 0, a+b \leq 4$.

$$
\psi(t)=\max \{\phi(t, t, t, a t, b t), \phi(t, 0,0, t, 0), \phi(0,0, t, t, t), \phi(0, t, t, 0,0)\}<t .
$$

If $f(X)$ is complete then $F, G$ and $f$ have a unique common fixed point $z$ in $X$ such that $\{f z\}=\{z\}=F z=G z$.

Remark 3.3. Corollary 3.1 improves and generalizes Theorem 3 of Khan \& Kubiaczyk [7].

If we put $f=g=I$ (the identity mapping on $X$ ) in Theorem 3.1, we get the following:

Corollary 3.2. Let $F, G$ be two set valued mappings of a metric space $(X, d)$ into $B(X)$ such that for all $x, y$ in $X$ and $\phi \in \Phi$

$$
\delta(F x, G y) \leq \phi(d(x, F x), d(y, G y), d(x, G y), d(y, F x), d(x, y))
$$

where $\psi \in \Psi, t>0, a \geq 0, b \geq 0, a+b \leq 4$.

$$
\psi(t)=\max \{\phi(t, t, t, a t, b t), \phi(t, 0,0, t, 0), \phi(0,0, t, t, t), \phi(0, t, t, 0,0)\}<t,
$$

then $F$ and $G$ have a unique common fixed point $z$ such that $\{z\}=F z=G z$.

Remark 3.4. Corollary 3.2, improves and generalizes Theorem 1 of Khan \& Kubiaczyk [7].

For a set valued map $F: X \rightarrow B(X)$ (respectively a single valued map $f: X \rightarrow X, F_{F}$ (respectively $F_{f}$ ) will denote the set of fixed points of $F$ (respectively $f$ ).

Theorem 3.3. Let $F, G$ be two set valued mappings of a metric space $(X, d)$ into $B(X)$ and $f, g$ two self mappings of $X$. If condition (1) holds for all $x, y \in X$ then

$$
\left(F_{f} \cap F_{g}\right) \cap F_{F}=\left(F_{f} \cap F_{g}\right) \cap F_{G} .
$$


Proof. If $u \in\left(F_{f} \cap F_{g}\right) \cap F_{F}$. Then

$$
\begin{aligned}
\delta(u, G u) & \leq \delta(F u, G u) \\
& \leq \phi(0, \delta(u, G u), \delta(u, G u), 0,0) \\
& \leq \psi(\delta(u, G u))<\delta(u, G u),
\end{aligned}
$$

which gives $G u=\{u\}$. Thus $\left(F_{f} \cap F_{g}\right) \cap F_{F} \subseteq\left(F_{f} \cap F_{g}\right) \cap F_{G}$.

Similarly one can show that $\left(F_{f} \cap F_{g}\right) \cap F_{G} \subseteq\left(F_{f} \cap F_{g}\right) \cap F_{F}$.

Theorem 3.1 and Theorem 3.3 imply the following:

Theorem 3.4. Let $F, G$ be two set valued mappings of a metric space $(X, d)$ into $B(X)$ and $f, g$ two self mappings of $X$. Suppose the pairs $\left\{F_{1}, f\right\}$ and $\left\{F_{2}, g\right\}$ are weakly commuting of type $(K B)$ on $X$, further

$$
F_{1}(X) \subseteq g(X), \quad F_{2}(X) \subseteq f(X),
$$

and for all $x, y$ in $X$ and $\phi \in \Phi$

$$
\begin{aligned}
\delta\left(F_{n} x, F_{n+1} y\right) \leq \phi( & \delta\left(f x, F_{n} x\right), \delta\left(g y, F_{n+1} y\right), \\
& \left.\delta\left(f x, F_{n+1} y\right), \delta\left(g y, F_{n} x\right), d(f x, g y)\right)
\end{aligned}
$$

where $\psi \in \Psi, t>0, a \geq 0, b \geq 0, a+b \leq 4$.

$$
\psi(t)=\max \{\phi(t, t, t, a t, b t), \phi(t, 0,0, t, 0), \phi(0,0, t, t, t), \phi(0, t, t, 0,0)\}<t .
$$

If one of $f(X)$ or $g(X)$ is complete then $f, g$ and $\left\{F_{i}\right\}_{i \in N}$, have a unique common fixed point in $X$.

\section{REFERENCES}

[1] E. Barcz, Some fixed point theorems for multivalued mappings, Demonstratio Math, 16 (1983), 735-744.

[2] T.H. Chang, Fixed point theorems of contractive type set valued mappings, Math. Japonica, 38(4) (1993), 675-690.

[3] B. Fisher and S. Sessa, Two common fixed point theorems for weakly commutuing mappings, Periodica Math. Hungarica, 20(3) (1989), 207-218.

[4] M. Imdad and A. Ahmad, On common fixed point of mappings and set valued mappings with some weak condition of commutativity, Publ. Math. Debrecen, 44/1-2 (1994), 105-114.

[5] G. Jungck, Compatible mappings and common fixed points, Internat. J. Math. Math. Sci., 9 (1986), 771-779.

[6] G. Jungck and B.E. Rhoades, Some fixed point theorems for compatible maps, Internat. J. Math. \& Math. Sci., 16(3) (1993), 417-428. 
[7] M.S. Khan, I. Kubiaczyk, Fixed point theorems for point to set maps, Math. Japonica, 33(3) (1988), 409-415.

[8] I. Kubiaczyk and B. Deshpande, Noncompatibility, discontinuity in consideration of common fixed point of set and single valued maps, Southeast Asian Bull. of Math., accepted for publication.

[9] S. Kyzyska and I. Kubiaczyk, Fixed point theorems for upper semicontinuous and weakly- weakly upper semicontinuous multivalued mappings, Math. Japonica, 47(2) (1998), 237-240.

[10] R.P. Pant, Common fixed points of noncommuting mappings, J. Math. Anal. Appl., 188 (1994), 436-440.

[11] R.P. Pant, Common fixed point theorems for contractive maps, J. Math. Anal. Appl., 226 (1998), 251-258.

[12] R.P. Pant, Common fixed points of Lipschitz type mappings pair, J. Math. Anal. Appl., 240 (1999), 280-283.

[13] R.P. Pant, Discontinuity and fixed points, J. Math. Anal. Appl., 240 (1999), 284-269.

[14] H.K. Pathak, Y.J. Cho and Kang, Remarks on R-weakly commuting mappings and common fixed point theorems, Bull. Korean Math. Soc., 34 (1997), 247-257.

[15] S. Sessa, On a weak commutativity condition of mappings in fixed point considerations, Publ. Inst. Math. (Beograd), 32(46) (1982), 149-153.

[16] S. Sessa and M.S. Khan, Some remarks in best approximation theory, Math. J. Toyoma Univ., 17 (1994), 151-165.

[17] S.P. Singh and B.A. Meade, On common fixed point theorems, Bull. Austral. Math. Soc., 16 (1977), 49-53.

Bhavana Deshrande

Department of Mathematics

Govt. Arts and Science P.G. College

RATLAM-457001 (M.P.)

INDIA

Home address:

Bhavana DESHPANDE

"SUKHAKARTA"

90-Rajiv Nagar (Near Kasturba Nagar)

RATLAM-457001 (M. P.)

INDIA

E-mail address: bhavnadeshpande@yahoo.com 